\title{
Robust Control of Ground Resonance in Helicopters Including Structural Uncertainties
}

\author{
José A. Ignácio da Silva* Gustavo L. C. M. de Abreu** \\ Douglas D. Bueno*** \\ * Mechanical Engineering Departament, University of São Paulo \\ State, Ilha Solteira, SP, (e-mail: jose.i.silva@unesp.br). \\ ** Mechanical Engineering Departament, University of São Paulo \\ State, Ilha Solteira, SP, (e-mail: gustavo.abreu@unesp.br). \\ *** Departament of Mathematics, University of São Paulo State, Ilha \\ Solteira, SP, (e-mail:douglas@mat.feis.unesp.br).
}

\begin{abstract}
Ground Resonance (GR) in helicopters is a potentially catastrophic instability defined in certain rotor speed ranges. It can limit the helicopter operational envelope and become the design of this type of vehicle a difficult task. A limited number of works in literature introduces formulations to compute the controller gain to suppress robustly this phenomenon. Also, commonly, a control approach defines a feedback particularly to a specific rotor speed. In this context, this works introduces a new methodology to design a robust active control system to stabilize GR of helicopter in presence of structural uncertainties. The proposed approach can suppress this instability in all rotor speed range by using only one control gain and based on linear matrix inequalities (LMIs). The Lyapunov stability criteria is used and the unstable rotor speed is considered as an adictional uncertain parameter together with the lead-lag blade frequency to define an associated polytopic convex space. Using convex optimization a robust control gain is computed the all unstable rotor speed range is robustly stabilized. A numerical simulations are carried out to demonstrate the effectiveness of this methodology. The results confirm the viability of proposed approach to design controllers for this problem.
\end{abstract}

Keywords:

Ground Resonance in Helicopters; Operational envelope; Structural uncertaints; Polytopic convex space; Robust control gain.

\section{INTRODUCTION}

Ground Resonance (GR) in helicopters is potentially catastrophic instability commonly caused by the coalescence of the regressive rotor mode with the fuselage in-plane motion in certain rotor speed ranges (Coleman and Feingold, 1956). A classical analysis of this phenomenon was developed by Coleman and Feingold (1956) using a simple linear mechanical model. Despite its relative simplicity, the method accurately predicts the GR boundaries of instabilities for isotropic rotors (i.e., all blades similar among them). In addition, different studies involving GR phenomenology can be found in (Hammond (1974); Janowski and Tongue (1988); Gourc et al. (2016) and references therein). Recently, Sanches et al. (2013) investigated the robustnees of the model and conclude that the parametric variation of a helicopter parameter (i.e., ex: lead-lag blade frequency) can expand strongly the GR boundaries of instabilities.

GR occurrence implies to excessive vibrations which can lead a catastrophic destruction of a helicopter if they are

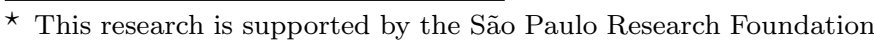
(FAPESP).
}

not reduced on time (Coleman and Feingold, 1956). Conventional approaches to mitigate this instability involve the installation of lag dampers on the blade root. These passive lag dampers are designed to work under particular conditions and their performance can be substantially reduced when they are applied on different situations. This is an important limitation for practical engineering applications. In another hand, the use of semi-active and active control strategies is an attractive option to suppression GR since they are capable of adjusting their dissipation levels for each rotor operational condition (da Silva et al., 2019).

Recent researches have employed semi-active techniques to suppress the GR vibrations, also using magnetorheological (MR) lag dampers. Gandhi et al. (2001) investigated the use of a MR damper with robust feedback linearization control law to alleviate the limit cycle instabilities including nonlinear effects. Zhao et al. (2004) studied the application of the on-off, sliding mode and feedback linearization control methodologies using MR dampers.

Although different methodologies in literature have been proposed to suppress ground resonance vibrations in helicopters, commonly they comprise control design approaches which define controllers gain for each particular 
steady rotor speed. However, this kind of instability typically occurs in a continuous range of rotation (Coleman and Feingold, 1956). In this sense, to consider a different control gain for each discrete rotor speed in a range can limit the use of active and semi-active techniques. The main difficult, besides designing several controller, is to switch the gain appropriately, which can involve unsafe procedures and require high computational capacity.

In this context, this article proposes the use of linear matrix inequalities (LMI) to compute only one control gain to stabilize a rotor speed range in which occurs ground resonance of helicopter. It is considered a parametric variation on the lead-lag blade frequency and the set of equations involving these uncertain parameters is easily described by convex polytopic hull in a GR boundary. An LMI version of the LQR- $\alpha$ (linear quadratic regulator) controller is used. Results and discussions based on the analytical developments and numerical simulations are introduced to show the proposed strategy to robust stabilize the GR.

After a brief introduction this paper is presented as follows: Section 2 presents the GR model. The robust GR analysis is developed in Section 3. Section 4 presents formulation of the robust controllers to suppress this instability. In Section 5 the simulation results involving the robust control of GR are presented. Finally, some concluding remarks are given in Section 6 .

\section{GROUND RESONANCE DYNAMIC MODEL}

The mechanical model used in this work captures the essential features of GR phenomenon. It is assumed that the helicopter on its landing gear can be represented by effective parameters applied at the rotor hub, as done in (Coleman and Feingold, 1956). The fuselage on the landing gear is considered isotropic, which can be represented by a rigid block with equivalent mass $m_{f}$ linked to linear springs with stiffness $k_{f}$ along the $x, z$ directions. It is further considered only inplane motions of the rotor hub, on the longitudinal $x$ and lateral $z$ directions, described by the $x_{f}$ and $z_{f}$ degrees of freedom, respectively.

The rotor-blades system is considered isotropic (all blades are similar among them), operating through constant angular rotor speed $(\Omega)$. It is composed by four blades $\left(N_{b}=\right.$ 4) symmetrically mounted on the rotor hub. Each blade presents the lead-lag motion $\xi_{k}$ (the blade in-plane motion) and its location is determined by the azimuthal angle $\psi_{k}$ $=\Omega t+\frac{\pi}{2}(k-1)$ with $x$-axis (Coleman and Feingold, 1956). The blades are considered uniform and rigid, also including the same mass $m_{b}$, mass moment $S_{b}$, and moment of inertia $I_{b}$ around the $y$-axis in relation to the lag rotating center (point A in Figure 2). They have a root linear torsional spring $k_{b}$ on the lag hinge. An illustrative scheme of this system is shown in Figures 1 and 2, where $e$ is the lag hinge offset, $b$ is the location of the blade's center of mass (C.M.) - the point B in Figure 2 measured from the lag hinge (point $\mathrm{A}$ in Figures 2). This illustration is an important scheme to simplify the understanding of the physical meaning behind the equations presented herein.

For this problem aerodynamic effects are not considered. Also, the fuselage and the blade structural damping are neglected as done in other similar self-excited vibration

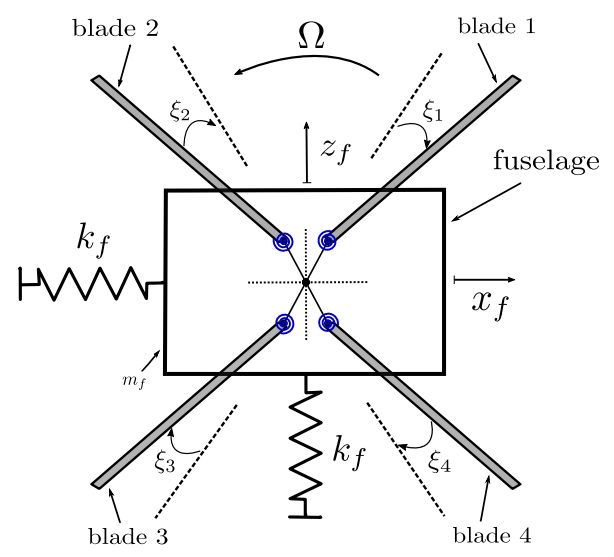

Figura 1. Mechanical model of a four-bladed helicopter rotor for GR analysis.

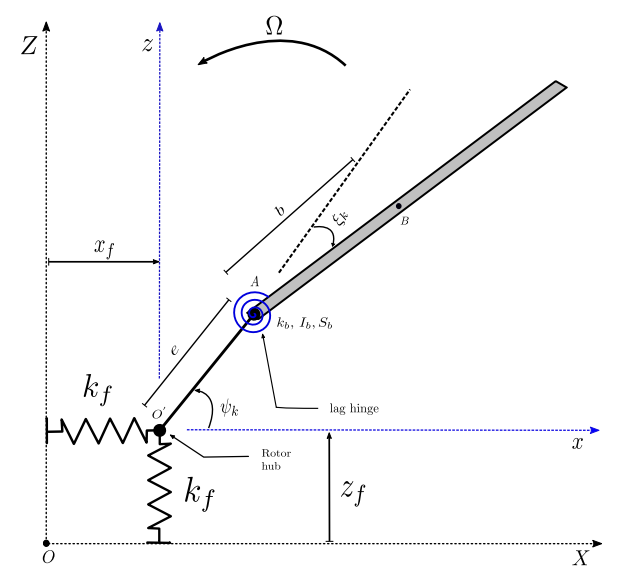

Figura 2. Detailed representation of the rotor-blade for a $k$-th blade.

control problems, as for an instance, the aeroelastic flutter (Bueno et al., 2015).

Based on the above description of the mechanical model, equations of motion for ground resonance is defined as,

$$
\begin{gathered}
\xi_{k}^{\prime \prime}+S x_{f}^{\prime \prime} \sin \left(\psi_{k}\right)-S z_{f}^{\prime \prime} \cos \left(\psi_{k}\right)+\left[\frac{\omega_{b}^{2}}{\Omega^{2}}+e S\right] \xi_{k}=0 \\
x_{f}^{\prime \prime}+\sum_{k=1}^{N_{b}} S_{f} \xi_{k}^{\prime \prime} \sin \left(\psi_{k}\right)+2 \sum_{k=1}^{N_{b}} S_{f} \xi_{k}^{\prime} \cos \left(\psi_{k}\right)- \\
-\sum_{k=1}^{N_{b}} S_{f} \xi_{k} \sin \left(\psi_{k}\right)+\frac{\omega_{f}^{2}}{\Omega^{2}} x_{f}=0 \\
z_{f}^{\prime \prime}-\sum_{k=1}^{N_{b}} S_{f} \xi_{k}^{\prime \prime} \cos \left(\psi_{k}\right)+2 \sum_{k=1}^{N_{b}} S_{f} \xi_{k}^{\prime} \sin \left(\psi_{k}\right) \\
+\sum_{k=1}^{N_{b}} S_{f} \xi_{k} \cos \left(\psi_{k}\right)+\frac{\omega_{f}^{2}}{\Omega^{2}} z_{f}=0
\end{gathered}
$$

where $\tau=\Omega t$ is nondimensional time, ()$^{\prime}=d() / d \tau=$ $d() / \Omega d t$ and ()$^{\prime \prime}=d^{2}() / d \tau^{2}=d^{2}() / \Omega^{2} d t^{2}$ indicate the first and second nondimensional time derivatives. $S=S_{b} / I_{b}$ is the normalized blade mass moment, $S_{f}=S_{b} / \tilde{M}_{f}$ are the normalized fuselage inertia, $\omega_{b}^{2}=k_{b} / I_{b}$ is the nonrotating lag blade frequency, $\omega_{f}^{2}=k_{f} / \tilde{M}_{f}$ are the fuselage frequency, with $\tilde{M}_{f}=m_{f}+N_{b} m_{b}$. In particular to this 
work, it is considered a nondimensional form of the model, where all the equation are divided by $\left(\Omega^{2}\right)$, as discussed in Section 4.1. This procedure is a classical approach used to study the GR problem, as shown in references (Coleman and Feingold (1956); Hammond (1974); Janowski and Tongue (1988); Zhao et al. (2004); Sanches et al. (2013); Gourc et al. (2016); and the references therein).

\subsection{Coleman Transformation}

GR system of equations is linear with periodic terms that depend on the azimuthal angle $\psi_{k}$ of each $k^{\text {th }}$ blade and it is (Johnson, 2013). In this case, classical stability analysis and linear control techniques are not suitable to deal with these periodic terms. Therefore, is convenient introduce the Coleman Transformation (CT) (Coleman and Feingold, 1956). CT is a mathematical tool that transforms the system of equations from a rotating frame (i.e., physical domain) to a non-rotating one (i.e., Coleman domain). In this second domain, the GR model is Linear Time Invariant (LTI) defined in function of the coupled rotor modes. For a four helicopter rotor-blades, the blade lag angle, $\xi_{k}$, can be written in terms of Coleman coordinates by (Johnson, 2013)

$$
\xi_{k}=\xi_{0}+\xi_{1 c} \cos \left(\psi_{k}\right)+\xi_{1 s} \sin \left(\psi_{k}\right)+(-1)^{k} \xi_{0^{-}}
$$

where the Coleman coordinates are defined by:

$$
\begin{gathered}
\xi_{0}=\frac{1}{N_{b}} \sum_{k=1}^{N_{b}} \xi_{k}, \quad \xi_{0^{-}}=\frac{1}{N_{b}} \sum_{k=1}^{N_{b}} \xi_{k}(-1)^{k} \\
\xi_{1 s}=\frac{2}{N_{b}} \sum_{k=1}^{N_{b}} \xi_{k} \sin \left(\psi_{k}\right), \xi_{1 c}=\frac{2}{N_{b}} \sum_{k=1}^{N_{b}} \xi_{k} \cos \left(\psi_{k}\right)
\end{gathered}
$$

The coordinates $\xi_{0}, \xi_{0^{-}}, \xi_{1 c}$ and $\xi_{1 s}$ represents the modes of the coupled rotor-blades system and the phisical meaning of these can be found in Coleman and Feingold (1956). Substituting (2) into (1a)-(1c) and considering the Coleman coordinates defined in $(3 a)-(3 b)$ a new system of equations can be defined. In particular a four-bladed helicopter, the rotor modes $\xi_{0}, \xi_{0^{-}}$are decoupled from other ones. The resulting system contains four degrees of freedom (dofs), i.e., the longitudinal and lateral hub dofs and the sine and cosine ciclic modes $\left(\xi_{1 c}\right.$ and $\left.\xi_{1 s}\right)$. In this case, the new set of equations can be compacted in a matrix form as

$$
\mathbf{M}_{n r} \mathbf{q}_{n r}^{\prime \prime}+\mathbf{G}_{n r} \mathbf{q}_{n r}^{\prime}+\mathbf{K}_{n r} \mathbf{q}_{n r}=\mathbf{0}
$$

where $\mathbf{q}_{n r}=\left\{\begin{array}{llll}x_{f} & z_{f} & \xi_{1 s} & \xi_{1 c}\end{array}\right\}^{T}$ is the vector of displacements (i.e., described in the non-rotating frame), $\mathbf{M}_{n r}, \mathbf{G}_{n r}$ and $\mathbf{K}_{n r}$ are respectively the mass, gyroscopic and stiffness matrices given by:

$$
\begin{aligned}
\mathbf{M}_{n r}= & {\left[\begin{array}{cccc}
1 & 0 & 2 S_{f} & 0 \\
0 & 1 & 0 & -2 S_{f} \\
S & 0 & 1 & 0 \\
0 & -S & 0 & 1
\end{array}\right], \mathbf{G}_{n r}=\left[\begin{array}{cccc}
0 & 0 & 0 & 0 \\
0 & 0 & 0 & 0 \\
0 & 0 & 0 & -2 \\
0 & 0 & 2 & 0
\end{array}\right] } \\
\mathbf{K}_{n r}= & {\left[\begin{array}{cccc}
\frac{\omega_{f}^{2}}{\Omega^{2}} & 0 & 0 & 0 \\
0 & \frac{\omega_{f}^{2}}{\Omega^{2}} & 0 & 0 \\
0 & 0 & \frac{\omega_{b}^{2}}{\Omega^{2}}+e S-1 & 0 \\
0 & 0 & 0 & \frac{\omega_{b}^{2}}{\Omega^{2}}+e S-1
\end{array}\right] }
\end{aligned}
$$

Based on the above matrices the following state-space realization can be used

$$
\mathbf{x}_{n r}^{\prime}=\mathbf{A}_{n r} \mathbf{x}_{n r}, \quad \text { with } \mathbf{A}_{n r}=\left[\begin{array}{cc}
\mathbf{0}_{4 \times 4} & \mathbf{I}_{4 \times 4} \\
-\mathbf{M}_{n r}^{-1} \mathbf{K}_{n r} & -\mathbf{M}_{n r}^{-1} \mathbf{G}_{n r}
\end{array}\right]
$$

where $\mathbf{x}_{n r}(\tau)=\left\{\begin{array}{ll}\mathbf{q}_{n r} & \mathbf{q}_{n r}^{\prime}\end{array}\right\}^{T} \in \mathbb{R}^{2 n \times 1}$ is the state vector, $\mathbf{A}_{n r} \in \mathbb{R}^{2 n \times 2 n}$ is the dynamic matrix and its eigenvalues allow to understand the system stability (Coleman and Feingold, 1956). $n=4$ is the number of dofs in the Coleman domain.

A parametric uncertain is included on the lead-lag blade frequency $\omega_{b}$, as the follow

$$
\omega_{b}=\omega_{b}(1+\delta)
$$

where $\delta$ corresponds to the parametrics relative uncertainties related to $\omega_{b}$, that can assume the values $\delta \in[-1,1]$.

\section{ROBUST GROUND RESONANCE ANALYSIS}

Using the dynamic matrix $\left(\mathbf{A}_{n r}\right)$ the robust ground resonance can be identified by studying the real part of its eigenvalues for each steady $\Omega$ in the operational helicopter envelope, considering the variation of the parameter $\delta$.

Tabela 1. Helicopter parameters used in the numerical application (Sanches et al., 2013).

\begin{tabular}{ll}
\hline Parameter & Value \\
\hline Number of blades, $N_{b}$ & 4 \\
Rotor Radius, $R$ & $5.2 \mathrm{~m}$ \\
Blade mass, $m_{b}$ & $31.9 \mathrm{~kg}$ \\
Blade mass moment, $S_{b}$ & $79.75 \mathrm{~kg} \cdot \mathrm{m}$ \\
Blade mass moment of inertia, $I_{b}$ & $458.375 \mathrm{~kg} \cdot \mathrm{m}^{2}$ \\
Lag hinge offset, $e$ & $0.2 \mathrm{~m}$ \\
Nominal lead-lag blade frequency, $\omega_{b_{0}}$ & $3 \mathrm{rad} / \mathrm{s}$ \\
Fuselage mass, $m_{f}$ & $2902.9 \mathrm{~kg}$ \\
Fuselage frequency, $\omega_{f}$ & $8 \mathrm{rad} / \mathrm{s}$ \\
\hline
\end{tabular}

Considering the helicopter parameters dated in the Table 1 , the robust GR boundary of instability is construted and presented in terms of modal damping in Fig. 3. It is detected an unstable system's behavior in range the $\Omega=$ $[\underline{\Omega}=3.9 \mathrm{~Hz}, \bar{\Omega}=5.9 \mathrm{~Hz}]$. In the analysis, the GR boundary can be divided in three parts, where $I, I I$ and $I I I$ refeer to the nominal $(\delta=0)$, upper $(\delta=1)$ and lower $(\delta=-1)$ bounds of the uncertain parameter. It is observed in $I I I$, that the system exibthis worst unstable behavior, where the range of intability is larger than in nominal condition and the instabilities are more agressive than others (i.e., the amplitudes of system's vibration grown up faster).

\section{CONTROLLER DESIGN TO SUPPRESS GR}

To provide the control action is considered a hypothetical actuator mounted on each blade. The interaction of the force with the actuator horn (See Figure 4) results in a control moment $\left(T_{a_{k}}\right)$ applied just in lag direction. A schematic illustration for the actuation attachment mechanism is shown in Figure 4 . Therefore, (1a) can be rewritten including the control action $\frac{T_{a_{k}}}{I_{b} \Omega^{2}}$ in its left hand side (i.e., instead of using zero). To write the control force vector in the non-rotating frame is necessary to consider 


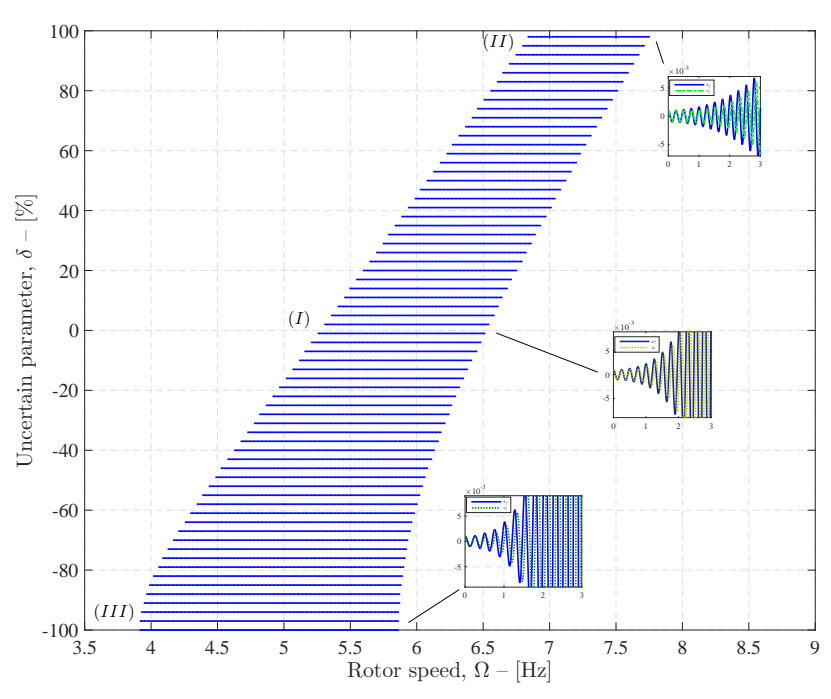

Figura 3. Robust Ground Resonance Boundaries

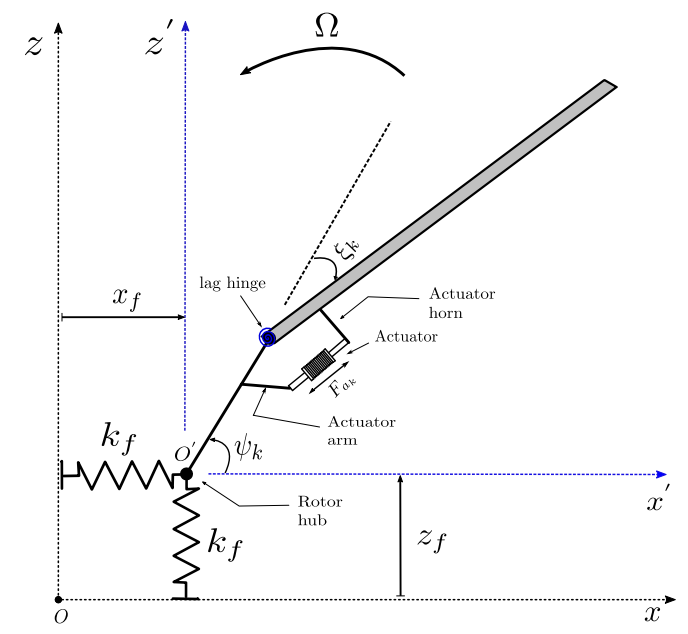

Figura 4. Attachment of the actuation's mechanism for GR mitigation.

the following $\mathrm{MBC}$ sine and cosine components of the control force

$$
T_{a_{1 s}}=\frac{2}{N_{b}} \sum_{k=1}^{N_{b}} T_{a_{k}} \sin \left(\psi_{k}\right), T_{a_{1 c}}=\frac{2}{N_{b}} \sum_{k=1}^{N_{b}} T_{a_{k}} \cos \left(\psi_{k}\right)
$$

In this case, the system of equations (4) can be rewritten also including the actuators force vector as

$$
\mathbf{M}_{n r} \mathbf{q}_{n r}^{\prime \prime}+\mathbf{G}_{n r} \mathbf{q}_{n r}^{\prime}+\mathbf{K}_{n r} \mathbf{q}_{n r}=\frac{\mathbf{T}_{a_{n r}}}{I_{b} \Omega^{2}}
$$

where $\mathbf{T}_{a_{n r}}=\left\{\begin{array}{lllll}0 & 0 & T_{a_{1 s}} & T_{a_{1 c}}\end{array}\right\}^{T}$ is the vector of control forces described in the non-rotating frame. The control input vector is defined by $\mathbf{u}_{n r}=\left\{T_{a_{1 s}} T_{a_{1 c}}\right\}^{T}$. The statespace realization of (9) results in

$\mathbf{x}_{n r}^{\prime}=\mathbf{A}_{n r} \mathbf{x}_{n r}+\mathbf{B}_{n r} \mathbf{u}_{n r}, \mathbf{B}_{n r}=\frac{1}{I_{b} \Omega^{2}}\left[\begin{array}{c}\mathbf{0}_{4 \times 2} \\ \mathbf{M}_{n r}^{-1} \tilde{\mathbf{B}}\end{array}\right], \tilde{\mathbf{B}}=\left[\begin{array}{ll}0 & 0 \\ 0 & 0 \\ 1 & 0 \\ 0 & 1\end{array}\right]$

where $\mathbf{B}_{n r} \in \mathbb{R}^{2 n \times m}$ is the control input matrix and $m$ is the number of control inputs.

\subsection{LMI formulation to LQR problem}

Consider a LTI system described by (10), the LQR-LMI controller problem consists to obtain a control gain $\mathbf{F} \in$ $\mathbb{R}^{m \times 2 n}$ such that $\mathbf{u}_{n r}=\mathbf{F} \mathbf{x}_{n r}$, that minimize the quadratic performance index

$$
J=\int_{0}^{\infty}\left(\mathbf{x}_{n r}^{T} \mathbf{Q} \mathbf{x}_{n r}+\mathbf{u}_{n r}^{T} \mathbf{R} \mathbf{u}_{n r}\right) d \tau
$$

where $\mathbf{Q}=\mathbf{Q}^{T}>\mathbf{0} \in \mathbb{R}^{2 n \times 2 n}$ and $\mathbf{R}=\mathbf{R}^{T}>\mathbf{0} \in$ $\mathbb{R}^{m \times m}$ are symmetric positive matrices that weighs the states and control energies, respectively (Boyd et al., 1994; Johnson and Erkus, 2006), and the pair $\left[\mathbf{A}_{n r}, \mathbf{B}_{n r}\right]$ must be controllable. The solution of LQR-LMI controller problem is introduced by Theorem 1 .

Theorem 1 The system described by (10) is asymptotically stable, in the sense of Lyapunov's theory, by a state feedback control law, $\mathbf{u}=\mathbf{F} \mathbf{x}$, guaranteed cost $J$, if exists the matrices $\mathbf{W}=\mathbf{W}^{T} \in \mathbb{R}^{2 n \times 2 n}, \mathbf{Z} \in \mathbb{R}^{m \times 2 n}$ and $\mathbf{X} \in \mathbb{R}^{2 n \times 2 n}$, such that satisfy the following convex minimization problem:

$$
\begin{aligned}
& \min \operatorname{Tr}(\mathbf{Q W})+\operatorname{Tr}(\mathbf{X}) \\
& \text { subject to } \\
& \mathbf{A}_{n r} \mathbf{W}+\mathbf{B}_{n r} \mathbf{Z}+\mathbf{W} \mathbf{A}_{n r}^{T}+\mathbf{Z}^{T} \mathbf{B}_{n r}^{T}<\mathbf{0} \\
& {\left[\begin{array}{cc}
\mathbf{X} & \mathbf{R}^{1 / 2} \mathbf{Z} \\
\mathbf{Z}^{T} \mathbf{R}^{1 / 2} & \mathbf{W}
\end{array}\right]>\mathbf{0}, \quad \mathbf{W}>\mathbf{0}}
\end{aligned}
$$

where the Lyapunov matrix and the state feedback gain are respectively given by $\mathbf{P}=\mathbf{W}^{-1}$ and $\mathbf{F}=\mathbf{W}^{-1} \mathbf{Z}$.

Proof: See Johnson and Erkus (2006).

The matrices $\mathbf{W}, \mathbf{X}$ and $\mathbf{Z}$ in (12) are obtained by solving the convex minimization problem and $\operatorname{Tr}()$ denotes the matrix trace.

\subsection{Polytopic Approach to GR Boundaries of Instability}

The main point in this article is to write the robust GR boundary of instability (see Figure 3 ) in terms of a polytopic convex hull. The strategy is to consider the rotor speed $\Omega$ and the lead-lag blade frequency $\omega_{b}$ as uncertainties parameters and define the associated polytopic convex hull. However, once the system of equations contains a quadratic $\left(\Omega^{-2}\right)$ and product terms $\left(\omega_{b}^{2} \Omega^{-2}\right)$ involving the uncertainties parameters, an algebraic manipulations must be previously used to write an LMI. In this sense, it is introduced the new uncertaints parameters $\theta=\Omega^{-2}$ and $\phi=\omega_{b}^{2} \Omega^{-2}$. Thence, it is considered the associated system $\left[\mathbf{A}_{n r}=\mathbf{A}_{n r}(\theta, \phi), \mathbf{B}_{n r}=\mathbf{B}_{n r}(\theta)\right]$ which depends on this new linear uncertainties parameters limited through the minimum $(\underline{())}$ and maximum $(\overline{()})$ values, i.e., $\theta \in[\underline{\theta}, \bar{\theta}]=$ $\left[\bar{\Omega}^{-2}, \underline{\Omega}^{-2}\right]$ and $\phi \in[\underline{\phi}, \bar{\phi}]=\left[0,4 \omega_{b_{0}}^{2} \underline{\Omega}^{-2}\right]$.

In practice, the admissible values of $\theta$ and $\phi$ are constrained in a polytope in the parameter space $\mathbb{R}^{n_{v}}$ with $n_{v}=2^{n_{r}}$ vertices (i.e., in this case $n_{r}=2$ ). This polytope is built by the convex combination of each vertex (Boyd et al., 1994). For this problem, the polytope of uncertain parameter is a rectangle with four vertices, $n_{v}=4$, in parameter space $\mathbb{R}^{2}$. Based on this values it is possible to define the polytopic system by

$$
\left[\mathbf{A}_{n r}, \mathbf{B}_{n r}\right](\theta, \phi) \in C o\left\{\left[\mathbf{A}_{n r}, \mathbf{B}_{n r}\right]_{i}, \ldots,\left[\mathbf{A}_{n r}, \mathbf{B}_{n r}\right]_{n_{v}}\right\} \equiv
$$




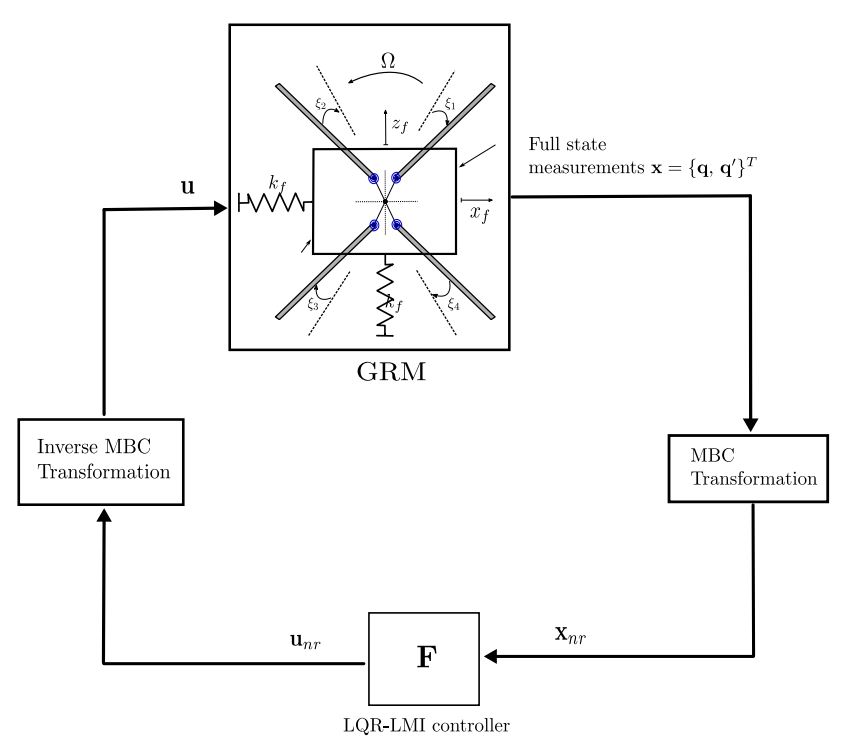

Figura 5. Feedback scheme for active suppression of GR phenomenon.

$$
\equiv\left\{\sum_{i=1}^{n_{v}} \lambda_{i}\left[\mathbf{A}_{n r}, \mathbf{B}_{n r}\right]_{i}, \sum_{i=1}^{n_{v}} \lambda_{i}=1, \lambda_{i} \geq 0\right\}, i=1, \ldots, n_{v}
$$

The introduced model nondimensionalization allows to reduce the polytope dimension. If the dimensional system is used, there are three terms to be defined as uncertain parameter $\left(\Omega\right.$ and $\Omega^{2}$ ). In this case, the polytope must be a cube (nine vertices) which can be more complex to be solved by the convex optimization algorithm in comparison with the approach solved herein.

To achieve a robust asymptotically stable system behavior in all GR boundary using an unique control gain it is necessary to write the LMI in (12) for each vertex of the polytope defined in (13), as given by the following LMI.

$$
\min [\operatorname{Tr}(\mathbf{Q W})+\operatorname{Tr}(\mathbf{X})] \text { subject to }
$$

$$
\begin{gathered}
\mathbf{A}_{i} \mathbf{W}+\mathbf{B}_{u i} \mathbf{Z}+\underset{i=1, \ldots, n_{v}}{\mathbf{W A}_{i}^{T}+\mathbf{Z}^{T} \mathbf{B}_{u i}^{T}+2 \alpha \mathbf{W}<\mathbf{0}} \\
{\left[\begin{array}{cc}
\mathbf{X} & \mathbf{R}^{1 / 2} \mathbf{Z} \\
\mathbf{Z}^{T} \mathbf{R}^{1 / 2} & \mathbf{W}
\end{array}\right]>\mathbf{0}, \quad \mathbf{W}>\mathbf{0}}
\end{gathered}
$$

The main advantage of describing this problem by convex polytopic hulls is the guarantee of achieving the robust stability in all rotor speed range where the GR occurs, using an unique control gain.

\subsection{Controller Implementation}

To compute the control gain is considered the new coordinate system obtained from the CT. Based on this gain, the control forces $\mathbf{u}_{n r}$ are defined in the non-rotating frame and they need to be converted to the physical coordinates for feedback practical applications. In this case, an inverse MBC transformation is applied on the control inputs, as pointed in da Silva et al. (2019) (see Figure 5).

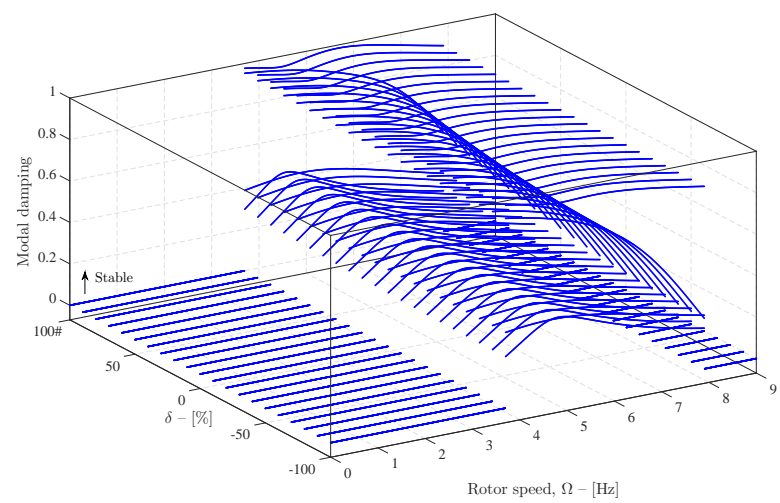

Figura 6. Robust Modal Damping Diagram for the controlled system.

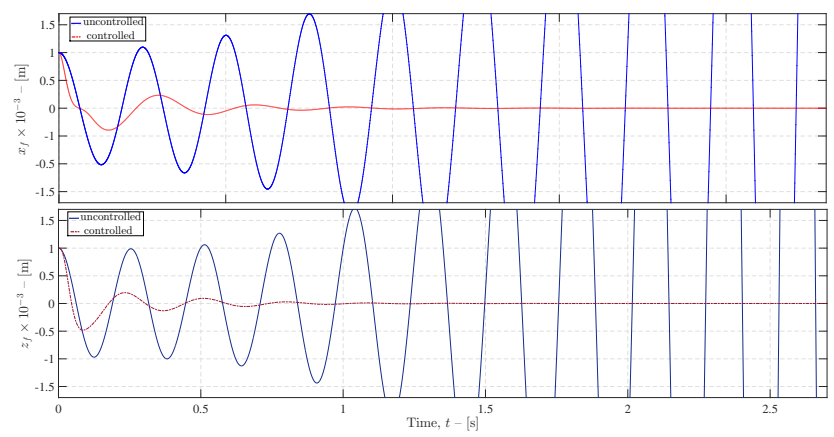

Figura 7 . Time response fuselage $\left(x_{f}\right.$ and $\left.z_{f}\right)$ motion in region $(I)$ at $(\Omega=5.7 \mathrm{~Hz})$.

\section{NUMERICAL APPLICATION}

To illustrate the proposed approach numerical simulations are carried out considering the helicopter parameters defined in Table 1. For this numerical application the control gains are computed by using $\mathbf{Q}=10^{-5} \times \mathbf{I}_{8 \times 8}$ and $\mathbf{R}=10^{3} \times \mathbf{I}_{2 \times 2}$, where $\mathbf{I}$ is the identity matrix. These values were defined arbitrarily.

Figure 6 shows the robust modal damping diagram for the controlled system. It is possible to note that values of modal damping are positive (i.e., equivalent to negative real parts eigenvalues of $\mathbf{A}_{n r}$ ) and the robust stability can be verified in all rotor speed range that defines a GR boundary.

Based on the controller designed, the time response of the system are evaluated in regions $(I),(I I)$ and $(I I I)$, in the rotor speeds correspondent to worst case of instability. The time responses are obtained by direct time integration of the system's equations of motion for the uncontrolled and controlled conditions. A fourth order Runge-Kutta method is used with initial condition $\mathbf{q}_{0}=$ $\left\{\begin{array}{llllll}0.001 & 0.001 & 0 & 0 & 0 & 0\end{array}\right\}^{T}$ and a time step equal to $10^{-4}$ seconds.

Figures 7, 8 and 9 show the worst longitudinal and lateral fuselage time response at regions $(I),(I I)$ and $(I I I)$. The results demonstrate the assured stability for each of these (i.e., the equilibrium position is reached in approx. 1.0 second), which demonstrate the robustnees and performance of the controller designed. 


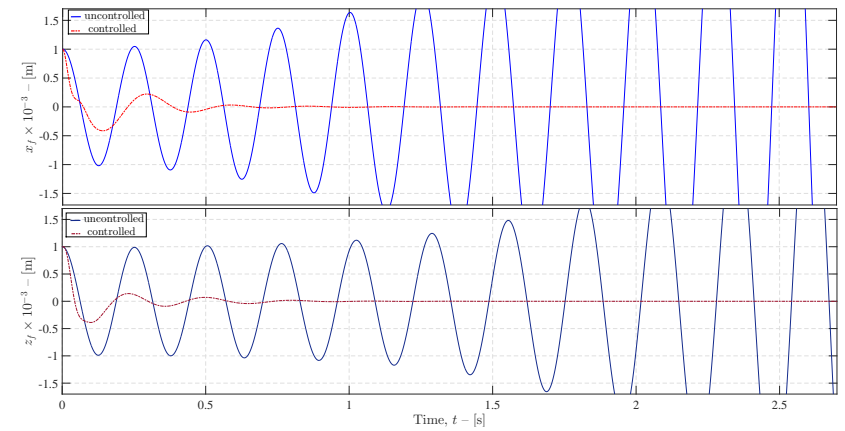

Figura 8. Time response fuselage $\left(x_{f}\right.$ and $\left.z_{f}\right)$ motion in region $(I I)$ at $(\Omega=7.0 \mathrm{~Hz})$.

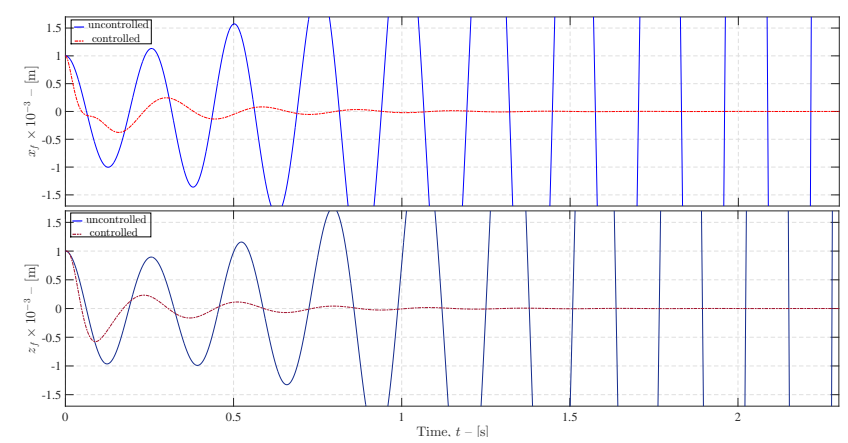

Figura 9. Time response fuselage $\left(x_{f}\right.$ and $\left.z_{f}\right)$ motion in region $(I I I)$ at $(\Omega=4.7 \mathrm{~Hz})$.
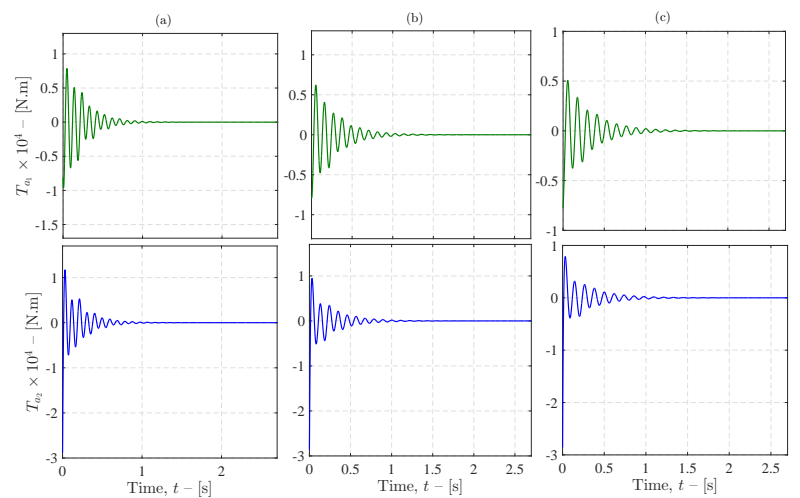

Figura 10. Control effort on the blades 1 and 2, (a) region $(I)$ at $(\Omega=5.7 \mathrm{~Hz}),(\mathrm{b})$ region $(I I)$ at $(\Omega=7.0 \mathrm{~Hz})$, (c) region $(I I I)$ at $(\Omega=4.7 \mathrm{~Hz})$.

The control efforts on the blades 1 and 2 for all regions ((I), (II) and (III)) are shown in Figure 10, where a maximum control effort of $30 \mathrm{kN} . \mathrm{m}$ is observed.

\section{FINAL CONCLUSIONS}

In this work an alternative approach to stabilize the GR phenomenon in helicopters in the presence of structural uncertainties using active control techniques and LMIs is presented. The classical linear mechanical model for GR analysis is employed. The main contribution of this work consists to represent the robust GR boundaries of instabilities (i.e., an unstable rotor speed range) by a convex polytopic hull. An LMI version of the LQR controller is employed. The quadratic stability criteria ensures that all previously unstable rotor speed range becomes asymptotically stable by using only one control gain. In particular, the proposed method is applied to solve the GR problem. Based on the present study, the following conclusions are obtained:

- The presence of structural uncertaints provide a representative expansion of the GR boundaries of instabilities.

- The approach used can suppress in totaly the GR phenomenon, demonstrateding that the idea in describing the robust GR boundaries by politopic convex spaces is an attractive solution for this problem.

\section{ACHNOLOGEMENTS}

The authors would like to thank the São Paulo Research Foundation (FAPESP), under Grant No. 2016/05988-2, for the financial support to this research.

\section{REFERENCIAS}

Boyd, S., V, V.B., Feron, E., and Ghaoui, L.E. (1994). Linear Matrix Inequalities in Systems and Control Theory, volume 15. Studies in Applied Mathematics, Society for Industrial and Applied Mathematics (SIAM).

Bueno, D.D., Goes, L.C.S., and Goncalves, P.J.P. (2015). Flutter analysis including structural uncertainties. Meccanica, 50(8), 2093-2101.

Coleman, R.P. and Feingold, A.M. (1956). Theory of selfexcited mechanical oscillations of helicopter rotors with hinged blades. techreport 1351, NACA.

da Silva, J.A.I., Bueno, D.D., and de Abreu, G.L.C.M. (2019). On the controllers design to stabilize ground resonance helicopter. Journal of Vibration and Control.

Gandhi, F., Wang, K.W., and Xia, L. (2001). Magnetorheological fluid damper feedback linearization control for helicopter rotor application. Smart Materials and Structures, 10(1), 96.

Gourc, E., Sanches, L., Michon, G., and Steffen, V. (2016). Post-critical analysis of ground resonance phenomenon: effect of stator asymmetry. Nonlinear Dynamics, 83(1), 201-215.

Hammond, C.E. (1974). An application of floquet theory to prediction of mechanical instability. Journal of the American Helicopter Society, 19(4), 14-23.

Janowski, M. and Tongue, B. (1988). Construction and analysis of a simplified non-linear ground resonance model. Journal of Sound and Vibration, 122(2), 233 241.

Johnson, E.A. and Erkus, B. (2006). Dissipativity and performance analysis of smart dampers via lmi synthesis. Structural Control and Health Monitoring, 14(3), 471-496.

Johnson, W. (2013). Rotorcraft Aeromechanics. Cambridge Aerospace Series. Cambridge University Press.

Sanches, L., Alazard, D., Michon, G., and Berlioz, A. (2013). Robustness analysis of helicopter ground resonance with parametric uncertainties in blade properties. Journal of Guidance, Control, and Dynamics, 36(1), $272-281$.

Zhao, Y., .Choi, Y.T., and Werely, N. (2004). Semiactive damping of ground resonance in helicopters using magnetorheological dampers. Journal of American Helicopter Society, 15, 468-492. 